\title{
Icariin stimulates osteogenic differentiation and suppresses adipogenic differentiation of rBMSCs via estrogen receptor signaling
}

\author{
XIAOYUN LI ${ }^{1}$, BOJIA PENG ${ }^{1}$, YANBIN PAN ${ }^{1}$, PANPAN WANG $^{1}$, KEHUAN SUN $^{2}$, XIAOTONG LEI $^{1}$, \\ LING OU $^{1}$, ZHIDI WU ${ }^{1}$, XIAOGUANG LIU ${ }^{2}$, HAIXIA WANG ${ }^{2}$, HAIBIN HE ${ }^{1}$, SHU MO ${ }^{2}$, \\ YA TIAN ${ }^{1}, X$ XUNQIAN PENG ${ }^{2}$, XIAOFENG ZHU ${ }^{3}$, RONGHUA ZHANG ${ }^{1}$ and LI YANG $^{1}$ \\ ${ }^{1}$ Department of Traditional Chinese Pharmacology, College of Pharmacy, Jinan University; \\ ${ }^{2}$ College of Traditional Chinese Medicine, Jinan University, Guangzhou, Guangdong 510632; \\ ${ }^{3}$ Department of The First Affiliated Hospital, Jinan University, Guangzhou, Guangdong 510630, P.R. China
}

Received January 23, 2018; Accepted June 19, 2018

DOI: $10.3892 / \mathrm{mmr} .2018 .9325$

\begin{abstract}
Icariin (ICA) is a major active ingredient in Herba epimedii, which is commonly used as a Chinese herbal medicine for the treatment of osteoporosis. Previous studies have revealed that ICA exerted a protective effect against bone loss and increased bone regeneration; however, the association between ICA and estrogen receptor (ER) signaling remains unclear. The aim of the present study was to determine the effect of ICA on rat bone marrow stromal cells (rBMSCs). Cell Counting Kit- 8 assays were conducted to measure proliferation, alkaline phosphatase (ALP) activity was evaluated to assess osteoblast differentiation, and reverse transcription-quantitative polymerase chain reaction as well as western blotting were performed to detect the expression of cellular and molecular markers of osteogenic or adipogenic differentiation. The results demonstrated that treatment of rBMSCs with $10^{-6} \mathrm{M}$ ICA stimulated rBMSC proliferation and ALP activity. Furthermore, ICA treatment increased the expression of the osteogenic markers runt-related transcription factor 2, collagen type 1 and bone morphogenetic Protein 2; however, it also decreased the expression of the adipogenic differentiation markers peroxisome proliferator-activated receptor gamma and CCAAT/enhancer-binding protein $\alpha$. Treatment of rBMSCs with ICI182780, an ER antagonist, blocked the effects of ICA. Taken together, these findings indicated that ICA may stimulate osteoblast differentiation and
\end{abstract}

Correspondence to: Professor Ronghua Zhang or Professor Li Yang, Department of Traditional Chinese Pharmacology, College of Pharmacy, Jinan University, 601 Huangpu Avenue West, Guangzhou, Guangdong 510632, P.R. China

E-mail: tzrh@jnu.edu.cn

E-mail: doctormonkey@126.com

Key words: icariin, estrogen receptor signaling, osteogenic differentiation, adipogenic differentiation inhibit adipogenic differentiation via the activation of the ER signaling pathway. Therefore, ICA has the potential to serve as a therapeutic alternative for the prevention and treatment of osteoporosis.

\section{Introduction}

There is a dynamic balance between osteoblast and adipocyte differentiation of bone marrow stromal cells (BMSCs). Under the action of signaling pathways, BMSCs have the potential to differentiate into osteoblasts or adipocytes (1). Postmenopausal osteoporosis (PMOP) is a skeletal disorder that results from osteoclastic bone resorption outpacing osteoblastic bone formation, is induced by estrogen deficiency, and characterized by decreased bone mass with an increased risk of fragility fracture (2). Current drugs available to treat PMOP aim to decrease osteoclastic activity or increase osteoblastic activity. Although estrogen replacement therapy is commonly used for PMOP prevention and treatment, it increases the risk of breast cancer and venous thromboembolism, and therefore, the risks outweigh the beneficial anti-resorptive effects of estrogen therapy (3). Denosumab, an androgen deprivation therapy, has reported side effects including sexual dysfunction, fatigue and metabolic syndrome (4). Bisphosphonates, the first-line anti-resorptive drug, have also been used to treat PMOP (5). However, due to the high incidence of osteonecrosis of the jaw and atypical femoral fractures in patients administered oral bisphosphonates for PMOP, such drugs cannot be routinely administered to patients in clinic (6). Therefore, there is a great need to seek novel therapeutic strategies and/or natural therapies that stimulate an anabolic effect on osteoporotic bone in PMOP patients.

The process of rat BMSC (rBMSC) differentiation involves various signaling pathways including MAPK and Notch pathway. Recently, plant-derived phytochemicals have received attention for their ability to induce osteoblast and osteoclast activity. Phytochemicals with promising therapeutic potential for osteoporosis include isoflavones, stilbenes and flavonoids (7). Icariin (ICA) is a major active flavonoid 
glycoside extracted from the Chinese herbal medicinal plant Epimedii, and it acts as a phytoestrogen. A study has shown that ICA protected against bone loss induced by ovariectomy or glucocorticoids (8). ICA treatment could increase osteogenic differentiation and bone formation of BMSCs in ovariectomized rat model of osteoporosis (9). In addition, ICA has recently been shown to play a vital role in the proliferation and osteogenic differentiation of rBMSCs and human BMSCs (10). Song et al (11) showed that ICA induced osteoblast differentiation through estrogen receptor (ER)-mediated activation of ERK and JNK signaling. Furthermore, this study showed that $17 \beta$-estradiol affects osteogenic and adipogenic differentiation through ER signaling (12). Therefore, we hypothesize that ICA may promote osteogenic differentiation of rBMSCs through an ER-mediated signaling pathway. ICI182780 (ICI) is a high affinity ER antagonist, which also acts as an agonist of the membrane-bound G-protein coupled ER (GPER).

In the experiments presented here, we used ICI as an ER antagonist. We investigate the effect of ICA on both osteogenic and adipogenic differentiation in rBMSCs, to further explore whether ICA regulates differentiation of rBMSCs via ER signaling.

\section{Materials and methods}

Cell culture. rBMSCs were purchased from Cyagen Biological Sciences (RASMX-01001; Santa Clara, CA, USA), and cultured in $\alpha$-MEM (Hyclone, Pittsburg, PA, USA) containing $10 \%$ fetal bovine serum (Tbd, China) in a humidified chamber with $5 \% \mathrm{CO}_{2}$ at $37^{\circ} \mathrm{C}$. The medium was replaced every three days.

Cell-counting kit-8 (CCK8) assay. rBMSCs were seeded into 96-well plates at a density of $5 \times 10^{3}$ cells/well, then treated with different concentrations of ICA $\left(10^{-4}, 10^{-5}, 10^{-6}, 10^{-7}\right.$, $10^{-8}, 10^{-9} \mathrm{M}$ ). After culturing for 24 or $48 \mathrm{~h}$, cell proliferation was measured using a CCK8 according to the manufacturer's instructions (Dojindo Laboratories, Kumamoto, Japan). Briefly, the culture medium was replaced with $100 \mu 1 \alpha$-MEM containing $10 \mu \mathrm{lCCK} 8$, and the plates were incubated for $1 \mathrm{~h}$ at $37^{\circ} \mathrm{C}$. Absorbance was measured at $450 \mathrm{~nm}$ using a multi-well spectrophotometer (BioTek, Synergy H4).

Alkaline phosphatase (ALP) activity. rBMSCs were seeded into 6-well plates at a density of $2 \times 10^{3}$ cells/well, and treated with different concentrations of ICA, as described in 2.2. Cells were treated with ICA for 3 and 7 days, and ALP activity assays were completed using ALP kits according to the manufacturer's instructions (Nanjing Jiancheng, Nanjing, China). Absorbance was measured at $520 \mathrm{~nm}$ using a multi-well spectrophotometer (BioTek, Synergy H4).

Alizarin red S staining. To determine calcium deposition, alizarin red staining was performed on day 7 of ICA treatment to evaluate the mineralized matrix. Cells were divided into control, $10^{-6} \mathrm{M}$ ICA and $\beta$-estradiol (E2) treatment groups. After culturing for 7 days, the medium was removed, cells were washed three times with PBS and fixed in 95\% ethanol for $10 \mathrm{~min}$, then washed twice with PBS, and stained with $0.1 \%$ alizarin red at a $\mathrm{pH}$ of 7.2 (Sigma). After incubating for
Table I. Primer sequences for reverse transcription-quantitative polymerase chain reaction.

\begin{tabular}{ll}
\hline Gene & \multicolumn{1}{c}{ Sequence (5'-3') } \\
\hline$E R \alpha$ & Forward: CATCGATAAGAACCGGAGGA \\
& Reserve: TCTGACGCTTGTGCTTCAAC \\
$E R \beta$ & $\begin{array}{l}\text { Forward: GAAGCTGAACCACCCAATGT } \\
\text { Reserve: CCAATCATGTGCACCAGTTC }\end{array}$ \\
$R U N X 2$ & Forward: CCACCACTCACTACCACACG \\
& Reserve: GGACGCTGACGAAGTACTAT \\
$B M P-2$ & Forward: GCCATCGAGGAACTTTCAGA \\
& Reverse: TGTTCCCGAAAAATCTGGAG \\
$P P A R \gamma$ & Forward: GGAATGCGTCATGAAAGGCG \\
& Reserve: GCGAACTTCAGTCCAGGTCA \\
$C / E B P \alpha$ & Forward: TTACAACAGGCCAGGTTTCC \\
& Reserve: CTCTGGGATGGATCGATTGT
\end{tabular}

ER, estrogen receptor; RUNX2, runt-related transcription factor 2; BMP-2, bone morphogenetic Protein 2; FABP, fatty acid-binding protein; $\mathrm{C} / \mathrm{EBP} \alpha, \mathrm{CCAAT} / \mathrm{enhancer}^{-b i n d i n g}$ protein $\alpha$; PPAR $\gamma$, peroxisome proliferator-activated receptor $\gamma$.

5-10 min at room temperature, cells were again washed three times with PBS and examined via light microscopy.

Oil red $O$ staining. rBMSCs were seeded into 6-well plates at a density of $2 \times 10^{3}$ cells/well, and divided into control, $10^{-6} \mathrm{M} \mathrm{ICA}$ and adipocyte-induced treatment groups. After rBMSCs were cultured for 14 days, oil red $\mathrm{O}$ staining was used to evaluate adipocyte differentiation of rBMSCs. Cells were rinsed twice with PBS, fixed with $4 \%$ paraformaldehyde for $15 \mathrm{~min}$, then rinsed twice more with PBS. treated with $60 \%$ isopropanol for 1 min, and infusion with oil red O (Sigma-Aldrich) that was dissolved in $60 \%$ isopropanol for $20 \mathrm{~min}$. This process was followed by three rinses with PBS. and then photographed.

Western blot analysis. rBMSCs were seeded into $10-\mathrm{cm}$ culture dishes and divided into control, ICA, ICI and ICI+ICA treatment groups. Total protein was extracted with RIPA lysis buffer (P0013B, Beyotime, Haimen, China). Total protein was separated with sodium dodecyl sulfate polyacrylamide gel electrophoresis and transferred onto polyvinylidene fluoride membranes. The membranes were washed in TBST, blocked with 5\% skim milk and incubated overnight with anti-ER $\alpha(1: 1,000$, ab133467, Abcam, USA) and anti-ER $\beta$ (1:1,000, ab32063, Abcam), anti-RUNX2 (1:1,000, 12556S, Cell Signaling Technology, Danvers, MA, USA), anti-BMP-2 (1:1,000, ab14933, Abcam), anti-PPAR $\gamma(1: 1,000, \mathrm{C} 26 \mathrm{H} 12$, Cell Signaling Technology), anti-CEBP/ $\alpha(1: 1,000,2295$, Cell Signaling Technology). Anti-GAPDH (1:1,000, D16H11, Cell Signaling Technology) was used as a loading control. The following day, membranes were incubated with anti-rabbit IgG secondary antibody $(1: 4,000,7074 \mathrm{P} 2$, Cell Signaling Technology). Immunoreactive bands were visualized with an electrochemiluminescence reagent (5312-1; DOCLAB, Guangzhou, China) and quantified using Image J (NIH). 

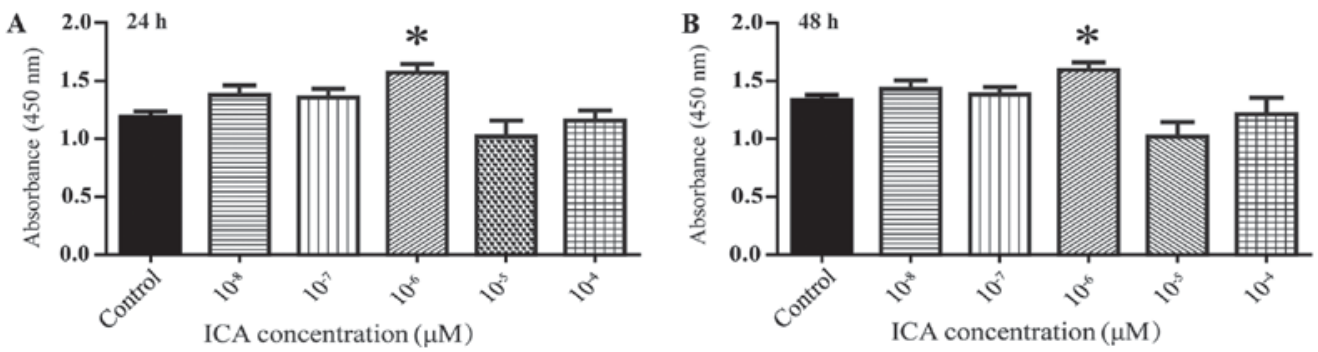

Figure 1. Effects of different concentrations of ICA on rBMSCs activity. rBMSCs were treated with different concentrations of ICA for (A) 24 or (B) 48 h. Data are presented as the mean \pm standard deviation $(n=3)$. ${ }^{*} \mathrm{P}<0.05$ vs. control. ICA, icariin; rBMSCs, rat bone marrow stromal cells.
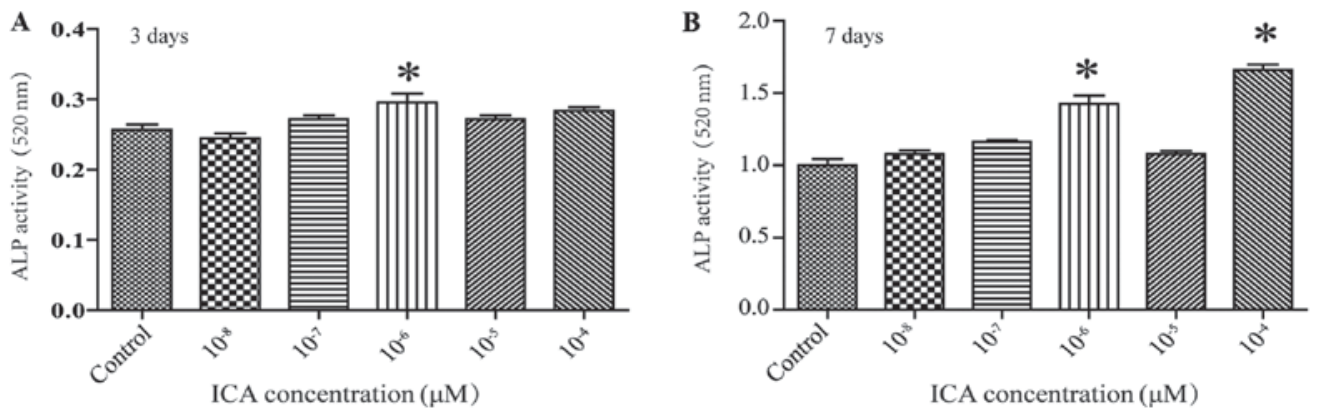

Figure 2. Effects of different concentration of ICA on ALP activity. Rat bone marrow stromal cells were treated with different concentrations of ICA for (A) 3 or (B) 7 days. Data are presented as the mean \pm standard deviation $(n=3)$. ${ }^{*} \mathrm{P}<0.05$ vs. control. ICA, icariin; ALP, alkaline phosphatase.

Reverse transcription-quantitative polymerase chain reaction (RT-qPCR). rBMSCs were seeded into 60-mm culture dishes, and divided into four groups, as aforementioned. Total RNA was isolated using TRIzol reagent (Invitrogen, Carlsbad, CA, USA) according to the manufacturer's instructions. cDNA was synthesized from mRNA using reverse transcriptase (AK4001; Takara, Shiga, Japan). After predenaturation at $95^{\circ} \mathrm{C}$ for $2 \mathrm{~min}, 40 \mathrm{RT}-\mathrm{qPCR}$ cycles were performed $\left(95^{\circ} \mathrm{C}\right.$ for $10 \mathrm{sec}$; and $60^{\circ} \mathrm{C}$ for $30 \mathrm{sec}$ ), followed by a final extension at $72^{\circ} \mathrm{C}$ for $10 \mathrm{~min}$. Target gene expression was calculated using the formula $2^{-\Delta \Delta \mathrm{Cq}}(13)$. Gene expression was normalized to $G A P D H$. Gene primer sequences are shown in Table I.

Statistical analysis. Data were collected from three separate experiments and expressed as the mean \pm standard deviation. The statistical differences were analyzed by one-way analysis of variance with Least Significant Difference and Student-Newman-Keuls post hoc tests using SPSS 19.0 software (IBM Corp., Armonk, NY, USA). P<0.05 was considered to indicate a statistically significant difference.

\section{Results}

Effects of different ICA concentrations on rBMSCs activity. CCK8 kits were used to measure cell activity. compared to control, treatment of rBMSCs with $10^{-6} \mathrm{M}$ ICA resulted in significantly increased cell activity at 24 and 48 h (Fig. 1; *P<0.05).

ICA promotes osteogenic differentiation of $r B M S C s$. ALP is a glycoprotein associated with the formation of calcified tissue, and it is the most widely recognized marker of the osteoblast phenotype. ALP activity is partially indicative of osteogenic differentiation. Treatment of cells with $10^{-6} \mathrm{M}$ ICA resulted in an increase in ALP activity by day of 3 of treatment, and this increase persisted through day 7 of treatment. (Fig. 2; $\left.{ }^{*} \mathrm{P}<0.05\right)$. ALP activity was also increased by $10^{-4} \mathrm{M}$ ICA on the seventh day (Fig. 2; ${ }^{*} \mathrm{P}<0.05$ ). These results, together with those detailed in section 3.1 , led us to choose a concentration of $10^{-6}$ ICA for the following experiments.

ICA promotes osteogenic differentiation. The number of mineralized nodules can serve as measure of osteogenic differentiation. Compare to control, the number of mineralized nodules was increased in cells treated with ICA, and was also increased cells treated with E2 (Fig. 3A-C). Fat droplets were identified by oil red $\mathrm{O}$ staining, and there was a reduction in the number and size of fat droplets in cells treated with ICA. (Fig. 3D-F; * $\mathrm{P}<0.05$ ).

ICA promotes the expression of osteogenic differentiation marker proteins and inhibits the expression of adipose differentiation marker proteins. Runt-related transcription factor 2 (RUNX2) and collagen type 1 (COL1) are markers of osteogenic differentiation, whereas peroxisome proliferator-activated receptor gamma (PPAR $\gamma$ ) and CCAAT/enhancer-binding protein alpha $(\mathrm{C} / \mathrm{EBP} \alpha)$ are important indicators of adipose differentiation. Compare with control group, the expression levels of ER $\alpha$ and ER $\beta$ proteins were significantly decreased with ICI treatment (Fig. 4). Treatment of cells with ICA, resulted in a significant decrease in PPAR $\gamma$ and $\mathrm{C} / \mathrm{EBP} \alpha$ protein expression, and a significant increase in $\mathrm{ER} \alpha, \mathrm{ER} \beta$, RUNX2 protein expression. However, compare to ICA treatment alone, when combined treatment of ICI+ICA, resulted in a significant increase in PPAR $\gamma$ protein expression and a significant decrease in RUNX2 and COL1 protein expression. 

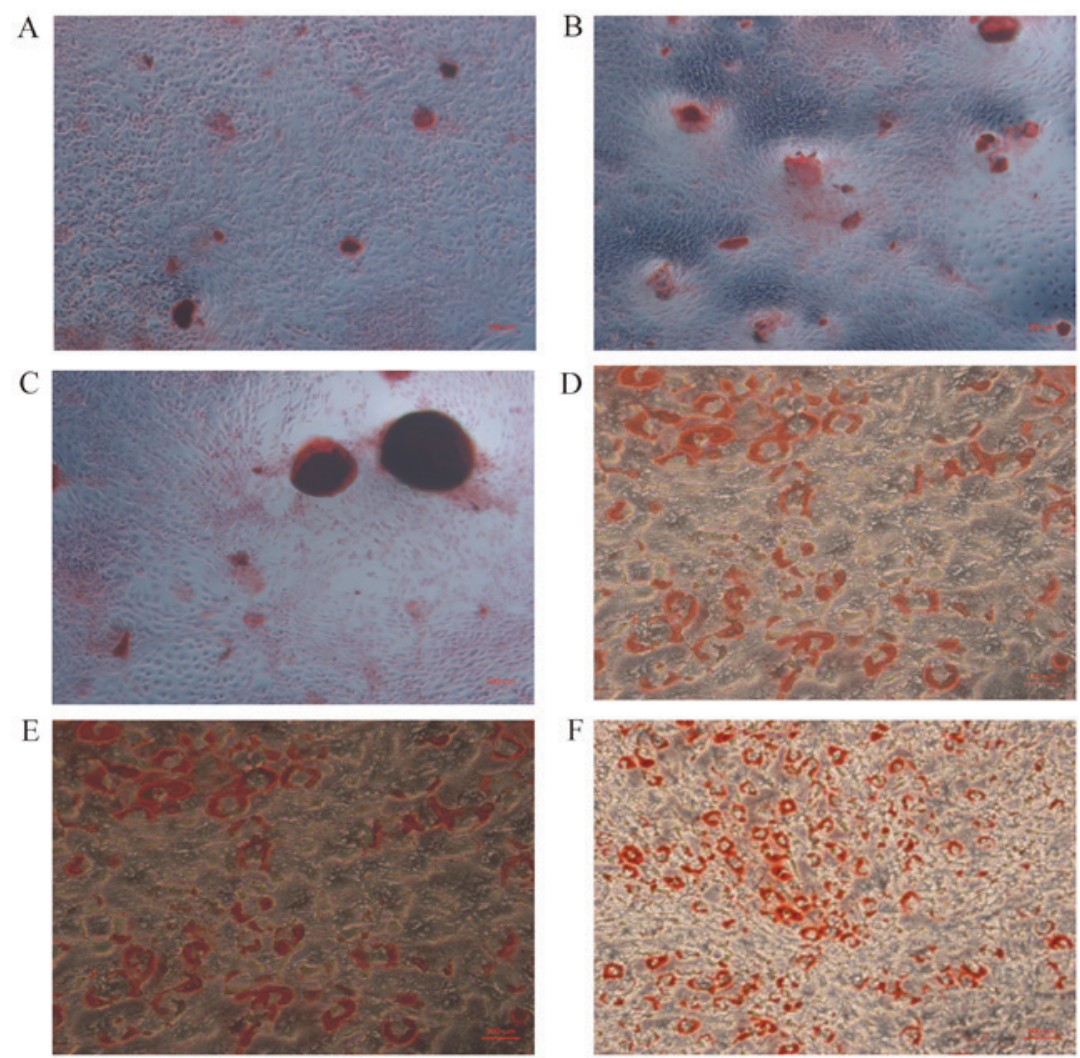

Figure 3. Alizarin red and Oil red O staining. Alizarin red staining of (A) control, (B) 10-6 M ICA and (C) E2 treated cells. Oil red O staining of (D) control, (E) $10^{-6}$ M ICA, and (F) adipocyte-induced cells (magnification, x200). ICA, icariin

While $\mathrm{C} / \mathrm{EBP} \alpha$ protein expression was not significantly different (Fig. 4; ${ }^{*} \mathrm{P}>0.05$ ).

ICA promotes the expression of osteoblast-specific genes and inhibits the expression of adipose-specific genes. Treatment of cells with ICI resulted in a significant decrease in ERQ and $E R \beta$ gene expression, (Fig. 5); treating cells with ICA resulted in a significant increase in the gene expression of bone morphogenic protein-2 (BMP-2) and runx2 compared to control (Fig. 5). These genes expression changes are in accordance with the changes of protein expression detected with Western-blot, suggesting that ICA stimulates osteogenic differentiation of rBMSCs by upregulating runx 2 and bmp-2 expression. In addition, ppar $\gamma$ and clebp $\alpha$ mRNA expression were decreased following treatment with ICA, suggesting that ICA acts to inhibit adipogenic differentiation. However, compared with ICA treatment, ppar $\gamma$ and c/ebp $\alpha$ were significantly increased, whereas bmp-2 and runx2 expression were significantly decreased with combined treatment of ICI+ICA. (Fig. 5; ${ }^{*} \mathrm{P}<0.05$ ).

\section{Discussion}

ER regulates physiological functions in almost all tissues in both males and females. Recent studies have shown that ER signaling plays an important role in many bone metabolism diseases (14). The classic ERs include ER $\alpha$ and ER $\beta$. ER $\alpha$ is predominantly expressed in cortical bone, whereas ER $\beta$ shows higher levels of expression in cancellous bone (15). ER $\alpha$ signaling activation regulates matrix mineralization in vitro, and its deficiency may lead to osteoporosis (16), ER $\beta$ signaling activation induces osteogenic differentiation of MC3T3-E1 cells and upregulates the expression of the osteogenesis-related factors including bone GLa protein and osteopontin. Previous study has indicated that ER signaling could regulate osteogenic and adipogenic differentiation (12). Therefore, in this study, we used ICI as an ER antagonist to determine whether ICA promotes osteogenic differentiation and inhibits adipogenic differentiation in an ER-dependent fashion. We demonstrated that ICI significantly downregulated ER $\alpha$ and ER $\beta$ protein and gene expression, suggesting that ICI successfully blocks ER-mediated signaling.

Bone metabolism is a balance between osteoblast-driven bone formation and osteoclast-mediated bone resorption. Osteogenic differentiation and adipogenic differentiation of rBMSCs are mutual inhibitory processes (11). Previous studies have demonstrated that ICA promotes osteogenic differentiation of MC3T3-E1 cells (17) and rBMSCs (18). Treatment with $10^{-6} \mathrm{M}$ ICA significantly increased rBMSCs activity, demonstrating that ICA promotes rBMSCs proliferation. Alizarin red $\mathrm{S}$ staining was used to visualize mineral deposition, and adipocyte accumulation was assessed with oil red $\mathrm{O}$ staining. The number of mineralized nodules was significantly increased with ICA treatment; while the number of fat droplets was significantly decreased, suggesting that ICA induces osteogenic differentiation but inhibits adipogenic differentiation. ALP is an early marker of osteoblast differentiation, and a peak in ALP activity is indicative of osteogenic differentiation (19). Treatment of $10^{-6} \mathrm{M}$ ICA significantly increased ALP activity by the third day of treatment, and ALP activity remained elevated on the seventh day of treatment. These 

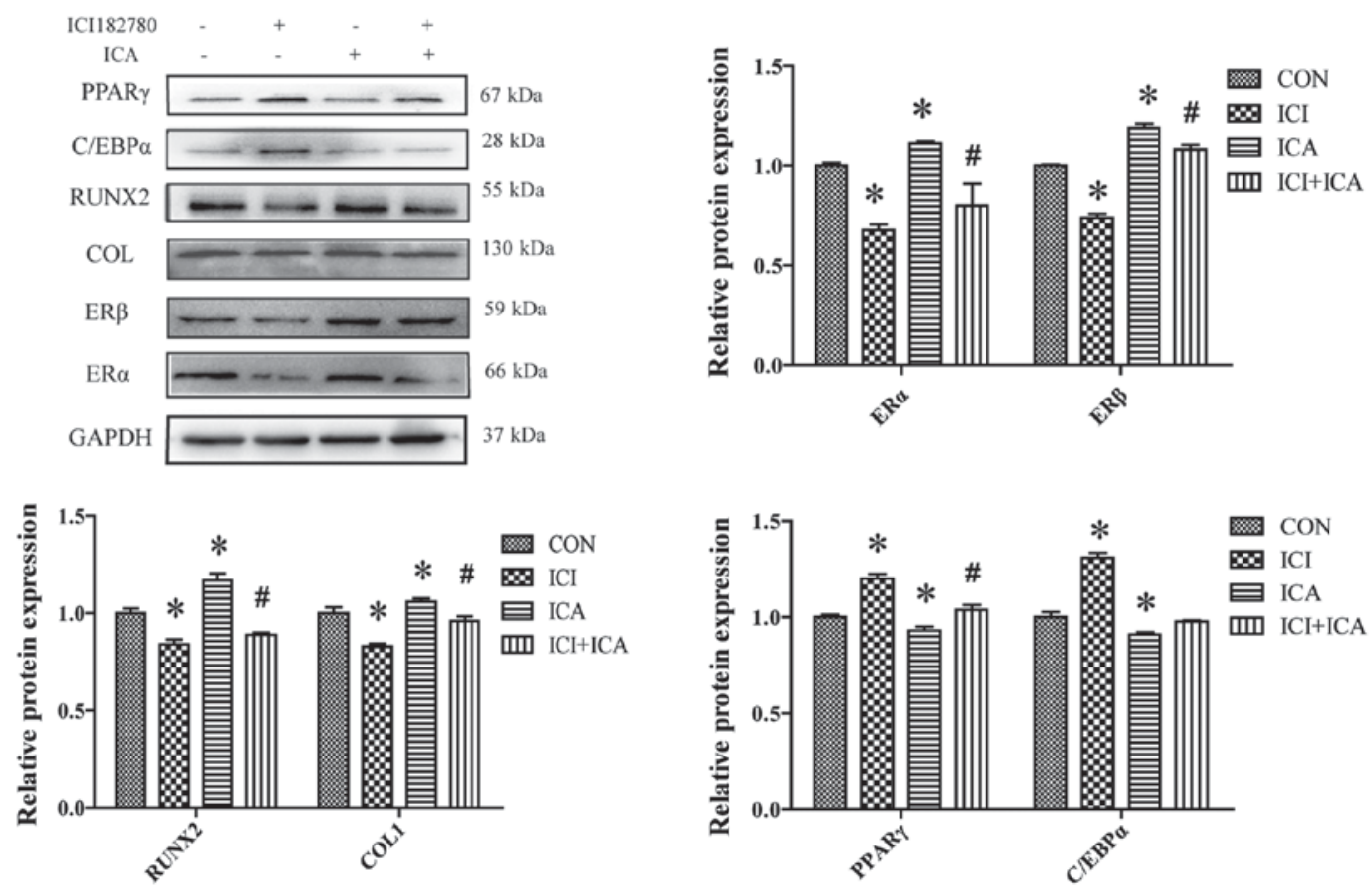

Figure 4. Western blot analysis of ER $\alpha, \mathrm{ER} \beta, \mathrm{RUNX} 2, \mathrm{COL} 1, \mathrm{C} / \mathrm{EBP} \alpha$ and PPAR $\gamma$ protein expression in rBMSCs treated with ICA and/or ICI. ICA treatment resulted in the downregulation of $\mathrm{C} / \mathrm{EBP} \alpha$ and PPAR $\gamma$ expression, and upregulation RUNX2 and COL1 expression. All of the observed effects were blocked by ICI treatment, except for $\mathrm{C} / \mathrm{EBP} \alpha$ expression. Data are presented as the mean \pm standard deviation $(\mathrm{n}=3){ }^{*} \mathrm{P}<0.05$ vs. control; ${ }^{\mathrm{P}} \mathrm{P}<0.05$ vs. ICA. ICA, icariin; ICI, ICI182780; ER, estrogen receptor; RUNX2, runt-related transcription factor 2; COL1, collagen type 1; PPAR $\gamma$, peroxisome proliferator-activated receptor- $\gamma$; $\mathrm{C} / \mathrm{EBP} \alpha, \mathrm{CCAAT} / \mathrm{enhancer-binding} \mathrm{protein} \alpha$; rBMSCs, rat bone marrow stromal cells; CON, control.
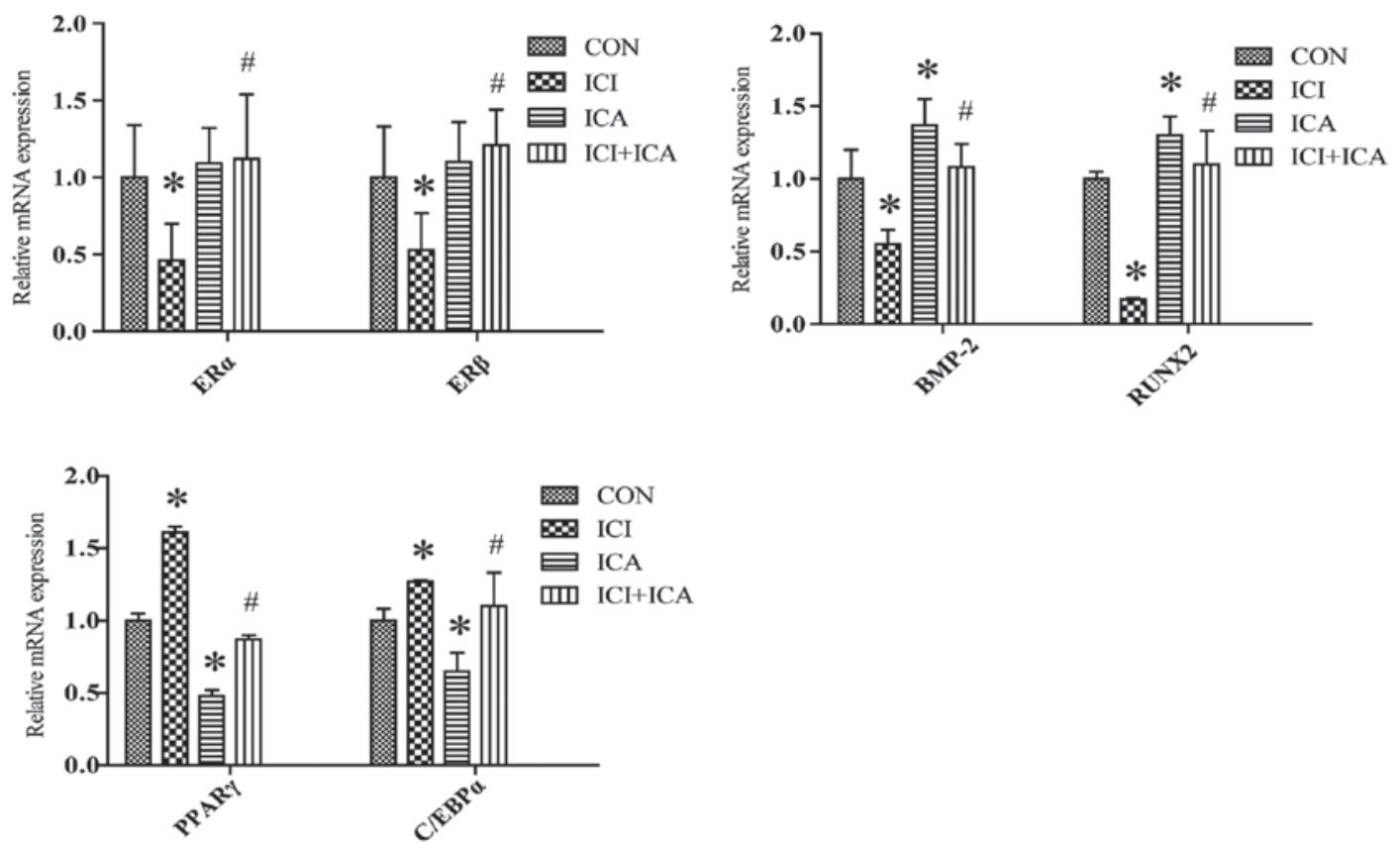

Figure 5. Reverse transcription-quantitative polymerase chain reaction analysis of $E R \alpha, E R \beta, R U N X 2, B M P-2, C / E B P \alpha$ and $P P A R \gamma$ gene relative expression in rat bone marrow stromal cells treated with ICA or ICI. Data are presented as the mean \pm standard deviation $(\mathrm{n}=3)$. " $\mathrm{P}<0.05$ vs. control; ${ }^{*} \mathrm{P}<0.05$ vs. ICA. ICA, icariin; ICI, ICI182780; ER, estrogen receptor; RUNX2, runt-related transcription factor 2; BMP-2, bone morphogenetic Protein 2; PPAR $\gamma$, peroxisome proliferator-activated receptor- $\gamma ; \mathrm{C} / \mathrm{EBP} \alpha, \mathrm{CCAAT} /$ enhancer-binding protein $\alpha$.

findings correspond to those from a previous study, which showed that ICA stimulated osteogenic differentiation (20) and inhibited adipocyte differentiation of rBMSCs (21).

RUNX2 acts as a scaffold for nucleic acids and regulatory factor involved in skeletal gene expression, and shows significantly increased expression in osteogenic differentiation (22); RUNX2 along with COL1, another important molecular marker of bone formation and bone remodeling, represent the foundation for matrix mineralization and have been shown to have increased expression or synthesis during 
osteogenic differentiation (23). BMP-2 plays a pivotal role in growth and differentiation. Huang et al (24) demonstrated that BMP-2 overexpression significantly stimulated osteocalcin and ALP gene expression. ICA treatment significantly upregulated the protein expression of RUNX2 and COL1 and the gene expression of runx2 and bmp-2. Together, these data suggest that ICA can promotes osteogenic differentiation. C/EBP $\alpha$ and PPAR $\gamma$ are the two molecules mostly likely to influence the regulation of adipocytes (25). In this study, the protein expression of PPAR $\gamma$ was significantly downregulated, as well as the genes expression of ppary and clebpa, suggesting that treatment with ICA can inhibit the adipogenic differentiation of rBMSCs.

Our previous study has shown that ICA can improve osteoporosis via the Notch signaling pathway (21), as well as the MAPK signaling pathway has also been shown to be involved in ICA-mediated osteogenic differentiation (26). In the present study, we use ICI as an ER signaling pathway antagonist, to investigate the correlation between ICA and the ER signaling pathway. The results showed that ICA treatment significantly upregulate the expression of $\mathrm{ER} \alpha$ and $\mathrm{ER} \beta$, suggesting that ICA may function by regulating ER signaling. Compared with ICA treatment alone, combined treatment with ICI+ICA resulted in a significant decrease in RUNX2 and COL1 protein expression, and a significant increase in PPAR $\gamma$, but there is no change in $\mathrm{C} / \mathrm{EBP} \alpha$ protein expression. Furthermore, genes expression of runx 2 and bmp-2 were also significantly decreased, while ppar $\gamma$ and c/ebp $\alpha$ were significantly with combined treatment increased. Together, these data suggest that the effects of ICA are blocked by ICI. Tao et al (27) have shown that prenylated flavonols can act as ER modulators. Taken together, these data demonstrate that ICA stimulates osteogenic differentiation and inhibits adipogenic differentiation via activation of ER signaling.

In conclusion, ICA promotes proliferation of rBMSCs, stimulates the osteogenic differentiation and mineralization of rBMSCs by regulating RUNX2, COL1 and BMP-2 expression, and inhibits adipogenic differentiation of rBMSCs by decreasing the expression of PPAR $\gamma$ and C/EBP $\alpha$. These effects can be blocked by ICI, which suggests that ICA stimulates osteogenic differentiation and inhibits adipocyte differentiation via activation of ER signaling.

\section{Acknowledgements}

The authors would like to thank the Cancer Research Institution of Jinan University (Shandong, China) for their contribution to the study.

\section{Funding}

The present study was supported by the National Natural Science Foundation of China (grant nos. 81473509 and 81673837), the National Natural Fund Youth Science Fund Project (no. 81503384) and the Fundamental Research Funds for the Central Universities.

\section{Availability of data and materials}

The datasets used and/or analyzed during the current study are available from the corresponding author on reasonable request.

\section{Authors' contributions}

RZ and LY conceived and designed the present study. XyL and BP performed western blotting, reverse transcriptionquantitative polymerase chain reaction and Alizarin S staining, and wrote the manuscript. YP completed CCK8 counting and data analysis. PW provided the cell line and performed cell culture. XtL and KS analyzed the western blotting data. LO and ZW performed the ALP activity experiments. XgL and HW conducted complete Oil red staining. HH, SM, YT, XP and $\mathrm{XZ}$ designed the structure of the article, performed the literature review, and critically revised the manuscript for important intellectual content.

\section{Ethics approval and consent to participate}

Not applicable.

\section{Patient consent for publication}

Not applicable.

\section{Competing interests}

The authors declare that they have no competing interests.

\section{References}

1. Berendsen AD and Olsen BR: Osteoblast-adipocyte lineage plasticity in tissue development, maintenance and pathology. Cell Mol Life Sci 71: 493-497, 2014.

2. Devlin MJ and Rosen CJ: The bone-fat interface: Basic and clinical implications of marrow adiposity. Lancet Diabetes Endocrinol 3: 141-147, 2015.

3. Eriksen EF, Díez-Pérez A and Boonen S: Update on long-term treatment with bisphosphonates for postmenopausal osteoporosis: A systematic review. Bone 58: 126-135, 2014.

4. Nguyen PL, Alibhai SM, Basaria S, D'Amico AV, Kantoff PW, Keating NL, Penson DF, Rosario DJ, Tombal B and Smith MR: Adverse effects of androgen deprivation therapy and strategies to mitigate them. Eur Urol 67: 825-836, 2015.

5. Francis MD and Valent DJ: Historical perspectives on the clinical development of bisphosphonates in the treatment of bone diseases. J Musculoskelet Neuronal Interact 7: 2-8, 2007.

6. Gao B, Huang Q, Lin YS, Wei BY, Guo YS, Sun Z, Wang L, Fan J, Zhang HY, Han YH, et al: Dose-dependent effect of estrogen suppresses the osteo-adipogenic transdifferentiation of osteoblasts via canonical Wnt signaling pathway. PLoS One 9: e99137, 2014.

7. Zhang YL, Jiang JJ, Shen H, Chai Y, Wei X and Xie YM: Total flavonoids from Rhizoma Drynariae (Gusuibu) for treating osteoporotic fractures: Implication in clinical practice. Drug Design Dev Ther 11: 1881-1890, 2017.

8. Hu J, Mao Z, He S, Zhan Y, Ning R, Liu W, Yan B and Yang J: Icariin protects against glucocorticoid induced osteoporosis, increases the expression of the bone enhancer DEC1 and modulates the PI3K/Akt/GSK $\beta / \beta$-catenin integrated signaling pathway. Biochem Pharmacol 136: 109-121, 2017.

9. Wu Y, Cao L, Xia L, Wu Q, Wang J, Wang X, Xu L, Zhou Y $\mathrm{Xu} \mathrm{Y}$ and Jiang $\mathrm{X}$ : Evaluation of osteogenesis and angiogenesis of icariin in local controlled release and systemic delivery for calvarial defect in ovariectomized rats. Sci Rep 7: 5077 , 2017.

10. Fu S, Yang L, Hong $H$ and Zhang $R$ : Wnt/ $\beta$-catenin signaling is involved in the Icariin induced proliferation of bone marrow mesenchymal stem cells. J Tradit Chin Med 36: 360-368, 2016 (In Chinese).

11. Song L, Zhao J, Zhang X, Li H and Zhou Y: Icariin induces osteoblast proliferation, differentiation and mineralization through estrogen receptor-mediated ERK and JNK signal activation. Eur J Pharmacol 714: 15-22, 2013. 
12. Niada S, Giannasi C, Ferreira LM, Milani A, Arrigoni E and Brini AT: $17 \beta$-estradiol differently affects osteogenic differentiation of mesenchymal stem/stromal cells from adipose tissue and bone marrow. Differentiation 92: 291-297, 2016.

13. Livak KJ and Schmittgen TD: Analysis of relative gene expression data using real-time quantitative PCR and the 2(-Delta Delta C(T)) method. Methods 25: 402-408, 2001.

14. Martin A, Yu J, Xiong J, Khalid AB, Katzenellenbogen B, Kim SH, Katzenellenbogen JA, Malaivijitnond S, Gabet Y, Krum SA and Frenkel B: Estrogens and androgens inhibit association of RANKL with the pre-osteoblast membrane through post-translational mechanisms. J Cell Physiol 232: 3798-3807, 2017.

15. Bord S, Horner A, Beavan S and Juliet C: Estrogen receptors alpha and beta are differentially expressed in developing human bone. J Clin Endocrinol Metab 86: 2309-2314, 2001.

16. Li X, Song QS, Wang JY, Leng HJ, Chen ZQ, Liu ZJ, Dang GT and Song CL: Simvastatin induces estrogen receptor-alpha expression in bone, restores bone loss, and decreases ERo expression and uterine wet weight in ovariectomized rats. J Bone Miner Metab 29: 396-403, 2011.

17. An X, Ma K, Zhang Z, Zhao T, Zhang X, Tang B and Li Z: miR-17, miR-21, and miR-143 enhance adipogenic differentiation from porcine bone marrow-derived mesenchymal stem cells. DNA Cell Biol 35: 410-416, 2016.

18. Wei Q, Zhang J, Hong G, Chen Z, Deng W, He W and Chen MH: Icariin promotes osteogenic differentiation of rat bone marrow stromal cells by activating the $\mathrm{ER} \alpha-\mathrm{Wnt} / \beta$-catenin signaling pathway. Biomed Pharmacother 84: 931-939, 2016.

19. Stucki U, Schmid J, Hämmerle CF and Lang NP: Temporal and local appearance of alkaline phosphatase activity in early stages of guided bone regeneration. A descriptive histochemical study in humans. Clin Oral Implants Res 12: 121-127, 2001.
20. Wei QS, He MC, Chen MH, Chen ZQ, Yang F, Wang HB, Zhang $\mathrm{J}$ and $\mathrm{He} \mathrm{W}$ : Icariin stimulates osteogenic differentiation of rat bone marrow stromal stem cells by increasing TAZ expression. Biomed Pharmacother 91: 581-589, 2017.

21. Liu H, Xiong Y, Zhu X, Gao H, Yin S, Wang J, Chen G, Wang C, Xiang L, Wang P, et al: Icariin improves osteoporosis, inhibits the expression of PPAR $\gamma, \mathrm{C} / \mathrm{EBP} \alpha$, FABP4 mRNA, N1ICD and jagged1 proteins, and increases Notch 2 mRNA in ovariectomized rats. Exp Ther Med 13: 1360-1368, 2017.

22. Wang H, Jiang Z, Zhang J, Xie Z, Wang Y and Yang G: Enhanced osteogenic differentiation of rat bone marrow mesenchymal stem cells on titanium substrates by inhibiting Notch3. Arch Oral Biol 80: 34-40, 2017

23. Kim J, Lee HW, Rhee DK, Paton JC and Pyo S: Pneumolysin-induced autophagy contributes to inhibition of osteoblast differentiation through downregulation of $\mathrm{Sp} 1$ in human osteosarcoma cells. Biochim Biophys Acta 1861: 2663-2673, 2017.

24. Huang W, Rudkin GH, Carlsen B, Ishida K, Ghasri P, Anvar B, Yamaguchi DT and Miller TA: Overexpression of BMP-2 modulates morphology, growth, and gene expression in osteoblastic cells. Exp Cell Res 274: 226-234, 2002.

25. Li Y, Rong Y, Bao L, Nie B, Ren G, Zheng C, Amin R, Arnold RD, Jeganathan RB and Huggins KW: Suppression of adipocyte differentiation and lipid accumulation by stearidonic acid (SDA) in 3T3-L1 cells. Lipids Health Dis 16: 181, 2017.

26. Wu Y, Xia L, Zhou Y, Xu Y and Jiang X: Icariin induces osteogenic differentiation of bone mesenchymal stem cells in a MAPK-dependent manner. Cell Prolif 48: 375-384, 2015.

27. Tao ZR, Liu J, Jiang YM, Gong L and Yang B: Synthesis of prenylated flavonols and their potents as estrogen receptor modulator. Sci Rep 7: 12445, 2017. 